\title{
Spiral computed tomographic scanning of the chest with three dimensional imaging in the diagnosis and management of paediatric intrathoracic airway obstruction
}

\begin{abstract}
Mayer Sagy, Mehdi Poustchi-Amin, Laura Nimkoff, Peter Silver, Mark Shikowitz, John C Leonidas
\end{abstract}

\begin{abstract}
Background - The usefulness of spiral computed tomographic (CT) scans of the chest with three dimensional imaging (3D-CT) of intrathoracic structures in the diagnosis and management of paediatric intrathoracic airway obstruction was assessed.

Methods - A retrospective review was made of five consecutive cases (age range six months to four years) admitted to the paediatric intensive care unit and paediatric radiology division of a tertiary care children's hospital with severe respiratory decompensation suspected of being caused by intrathoracic large airway obstruction. Under adequate sedation, the patients underwent high speed spiral CT scanning of the thorax. Non-ionic contrast solution was injected in two patients to demonstrate the anatomical relationship between the airway and the intrathoracic large vessels. Using computer software, three-dimensional images of intrathoracic structures were then reconstructed by the radiologist.
\end{abstract}

Results - In all five patients the imaging results were useful in directing the physician to the correct diagnosis and appropriate management. In one patient, who had undergone repair of tetralogy of Fallot with absent pulmonary valve, the 3D-CT image showed bilateral disruptions in the integrity of the tracheobronchial tree due to compression by a dilated pulmonary artery. This patient underwent pulmonary artery aneurysmorrhaphy and required continued home mechanical ventilation via tracheostomy. In three other patients with symptoms of lower airway obstruction the 3D-CT images showed significant stenosis in segments of the tracheobronchial tree in two of them, and subsequent bronchoscopy established a diagnosis of segmental bronchomalacia. These two patients required mechanical ventilation and distending pressure to relieve their bronchospasm. In another patient who had undergone surgical repair of intrathoracic tracheal stenosis three years prior to admission the 3D-CT scan ruled out restenosis as the reason for her acute respiratory decompensation.
Conclusions - 3D-CT scanning is a useful additional diagnostic tool for intrathoracic airway obstruction in paediatric patients. (Thorax 1996;51:1005-1009)

Keywords: spiral CT scanning, three-dimensional imaging, children, airway obstruction.

Intrathoracic large airway obstruction is a clinical, diagnostic, and therapeutic challenge in paediatric emergency medicine. ${ }^{1}$ Some patients present to the emergency department with severe respiratory distress and wheezing, and the clinical differential diagnosis between reactive airway diseases such as asthma or bronchopulmonary dysplasia and intrathoracic large airway obstruction becomes important. While in acute asthma the treatment is primarily directed to bring about bronchodilation, in intrathoracic large airway obstruction this therapeutic approach may be of no avail.

Spiral or helical computed tomographic (CT) scanning offers certain technical advantages over the conventional CT scan as it is faster and more sensitive in detecting small lesions, ${ }^{2}$ and minimises motion and respiratory artifacts. This technique allows the production of overlapping images without additional radiographic exposure. By using specific computer software, high quality three dimensional images can be generated from the multiple overlapping transaxial images acquired in a single breath hold. ${ }^{3}$ When this technology is combined with intravenous injection of radiolabelled contrast media CT angiography is obtained.

Diagnostic efforts to identify possible obstruction of the intrathoracic airway include fluoroscopy, CT scanning, magnetic resonance imaging (MRI), bronchoscopy, bronchography, or any combination of these procedures. ${ }^{4}$ Among the non-invasive methods only spiral CT scanning with three dimensional imaging (3D-CT) provides three dimensional information. ${ }^{56}$ However, its usefulness in paediatrics in general, and in paediatric intrathoracic airway obstruction in particular, has not been fully explored. We have therefore evaluated the usefulness of this technology in five patients who were admitted to our paediatric intensive care unit with possible intrathoracic airway obstruction. 
Table 1 Clinical data

\begin{tabular}{|c|c|c|c|c|c|}
\hline Patient no. & Age & Diagnosis and symptoms & $3 D-C T$ findings & Other diagnostic tests & Management \\
\hline 1 & $18 \mathrm{~m}$ & $\begin{array}{l}\text { Tetralogy of Fallot and absent } \\
\text { pulmonary valve; wheezing and } \\
\text { increased requirement for oxygen } \\
\text { and ventilator settings }\end{array}$ & $\begin{array}{l}\text { Disruption in integrity of right upper } \\
\text { lobe bronchus, bronchus intermedius } \\
\text { and left lower lobe bronchus; severe } \\
\text { pulmonary artery dilation }\end{array}$ & $\begin{array}{l}\text { Chest radiography } \\
\text { Heart catheterisation }\end{array}$ & $\begin{array}{l}\text { Pulmonary aneurysmorrhaphy } \\
\text { Home mechanical ventilation }\end{array}$ \\
\hline 2 & $2 \mathrm{y}$ & $\begin{array}{l}\text { Chronic lung disease of prematurity; } \\
\text { refractory episodes of bronchospasm }\end{array}$ & $\begin{array}{l}\text { Disruption in integrity of left main stem } \\
\text { bronchus with a stenotic segment }\end{array}$ & $\begin{array}{l}\text { Chest radiography } \\
\text { Bronchoscopy }\end{array}$ & $\begin{array}{l}\text { Mechanical ventilation with } \\
\text { PEEP } 10 \mathrm{~cm} \mathrm{H}_{2} \mathrm{O} \text { for } 10 \text { days }\end{array}$ \\
\hline 3 & $4 \mathrm{y}$ & $\begin{array}{l}\text { Chronic lung disease of prematurity } \\
\text { and cardiomyopathy; repair of } \\
\text { tracheal stenosis; respiratory failure }\end{array}$ & $\begin{array}{l}\text { Normal configuration of bronchial tree; } \\
\text { irregular tracheal wall at site of surgery }\end{array}$ & $\begin{array}{l}\text { Chest radiography } \\
\text { Heart catheterisation }\end{array}$ & $\begin{array}{l}\text { Mechanical ventilation and } \\
\text { inotropic support }\end{array}$ \\
\hline 4 & $3 \mathrm{y}$ & $\begin{array}{l}\text { Marfan's disease; severe episodes of } \\
\text { bronchospasm }\end{array}$ & $\begin{array}{l}\text { Normal bronchial tree and mild dilation } \\
\text { of the ascending aorta }\end{array}$ & $\begin{array}{l}\text { Chest radiography } \\
\text { Echocardiography }\end{array}$ & Bronchodilators \\
\hline 5 & $6 \mathrm{~m}$ & $\begin{array}{l}\text { Brain damage, aspirations and } \\
\text { refractory bronchospasm }\end{array}$ & $\begin{array}{l}\text { Narrow segments in the left upper lobe } \\
\text { and right middle lobe bronchi }\end{array}$ & $\begin{array}{l}\text { Chest radiography } \\
\text { Bronchoscopy }\end{array}$ & $\begin{array}{l}\text { Tracheostomy for administration } \\
\text { of } 10 \mathrm{~cm} \mathrm{H} \mathrm{H}_{2} \mathrm{O} \text { CPAP }\end{array}$ \\
\hline
\end{tabular}

\section{Methods}

Five patients consecutively admitted with acute respiratory decompensation suspected to be secondary to intrathoracic large airway obstruction (table 1) were sedated and underwent high speed spiral CT scans of the chest ( $\mathrm{Hi}-$ Speed Advantage, General Electric, Milwaukee, Wisconsin, USA) with a $20-30$ second exposure time. Two patients received intravenous non-ionic radiocontrast medium prior to the CT scan for better delineation of their intrathoracic large vessels. Three dimensional images of intrathoracic structures were subsequently reconstructed by the radiologist using specific computer software (Advantage Window Computer Work Station, General Electric). Layers of tissue were sequentially removed, as deemed necessary, by successively changing the threshold level.

\section{PATIENT 1}

An 18 month old infant who had undergone surgery to repair tetralogy of Fallot with absent pulmonary valve. He had also required two additional surgical procedures to reduce the size of a dilated pulmonary artery which compressed the main stem bronchi and caused respiratory failure. From the age of five months the patient had been on home mechanical ventilation via a tracheostomy cannula. His most recent admission to the paediatric intensive care unit was secondary to inability to wean him off his home ventilator and the occurrence of frequent episodes of respiratory distress due to lower airway obstruction refractory to bronchodilators. Chest radiographs revealed a hyperinflated right lower lobe and repeat heart catheterisation revealed a massively dilated pulmonary artery. The $3 \mathrm{D}-\mathrm{CT}$ scan (fig $1 \mathrm{~A}$ and B) showed a marked disruption of the integrity of the right upper lobe bronchus, bronchus intermedius, and the left lower lobe bronchus. The patient underwent a third pulmonary artery aneurysmorrhaphy to relieve the compression on the airway. His bronchospasm subsided and he was discharged to be gradually weaned off his ventilator.

\section{PATIENT 2}

A two year old black patient was admitted to the paediatric intensive care unit because of severe bronchospasm and acute alveolar hypo- ventilation. His trachea was intubated and mechanical ventilation was initiated. His perinatal history revealed maternal oligohydramnios in association with fetal posterior urethral valves and severe obstructive damage to the kidneys. He was born after 28 weeks of gestation, required six weeks of mechanical ventilation, and developed bronchopulmonary
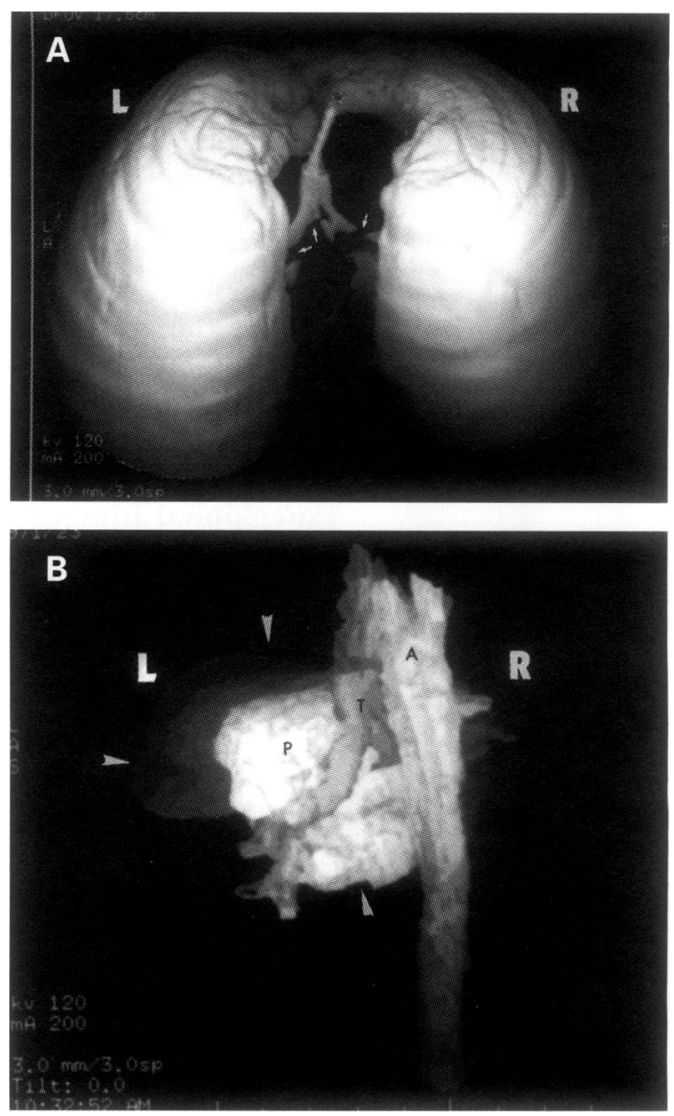

Figure 1 (A) Posterior view of a 3D-CT image of the chest in an 18 month old patient who had undergone repair of tetralogy of Fallot with absent pulmonary valve (patient 1). The arrows indicate disruptions in the tracheobronchial configuration (for normal configuration see fig 4A). These are caused by the dilated pulmonary artery compressing the left lower lobe bronchus and the right upper lobe bronchus and bronchus intermedius. Note that the tracheostomy cannula makes the upper portion of the trachea appear narrow because of differences between the radiographic density of the tracheal wall and the plastic cannula. (B) Further removal of intrathoracic tissue layers by changing density threshold levels to delineate the relationship between the tracheobronchial tree and the great vessels. The trachea $(T)$ is between a massively dilated main pulmonary artery $(P$, marked by arrows) and the aorta $(A)$. 


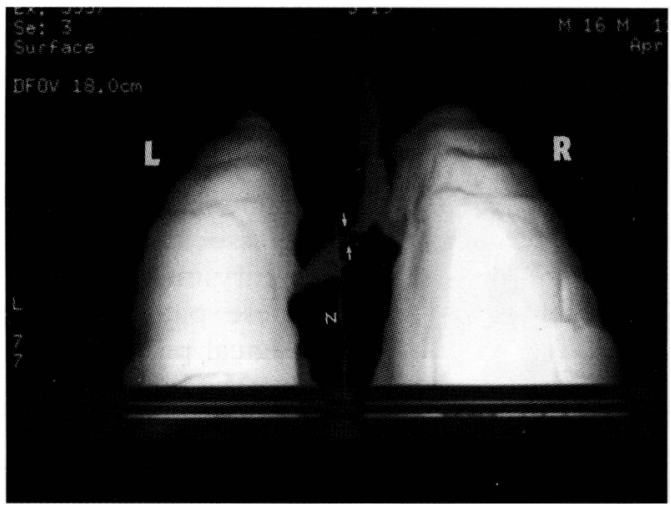

Figure 2 Posterior view of 3D-CT image of the lungs of a two year old patient with bronchopulmonary dysplasia and recurrent episodes of wheezing and respiratory distress (patient 2). The arrows indicate a stenotic segment in the left main stem bronchus (for normal configuration of tracheobronchial tree see fig 4A). The nasogastric tube is also depicted $(N)$. dysplasia (BPD). He has also been on chronic ambulatory peritoneal dialysis and is currently awaiting kidney transplantation. During the eight months before his admission he had multiple episodes of acute respiratory distress which resulted in endotracheal intubation and mechanical ventilation. His chest radiograph revealed persistent bilateral consolidations and a 3D-CT scan showed a stenosis of his left main stem bronchus (fig 2). Subsequent bronchoscopic examination confirmed the existence of a collapsed pliable segment in the left main bronchus compatible with a diagnosis of segmental bronchomalacia. Intraoperative administration of $10 \mathrm{~cm} \mathrm{H}_{2} \mathrm{O}$ of distending pressure dilated the segment and maintained it open. The patient received one week of mechanical ventilation with positive end expiratory pressure (PEEP) $10 \mathrm{~cm} \mathrm{H}_{2} \mathrm{O}$ and was gradually weaned off the ventilator. His parents refused chronic administration of CPAP via tracheostomy to prevent future similar episodes. The patient has been followed for six months since discharge during which time he has not had any episodes of respiratory discomfort. He was subsequently referred to another hospital for kidney transplantation.

PATIENT 3

A four year old white girl with chronic cardiomyopathy and BPD was admitted to the paediatric intensive care unit because of an increased requirement in her home ventilator settings and oxygen. The patient had undergone repair of double outlet right ventricle a few months after birth. At the age of one year, due to progressive respiratory distress, a tracheal stenosis at the level of the carina was diagnosed and repaired by resection and endto-end anastomosis. Her chest radiograph revealed bilateral pulmonary congestion and a 3D-CT scan delineated a normal configuration of the tracheobronchial tree with some irregularities at the level of her past tracheal

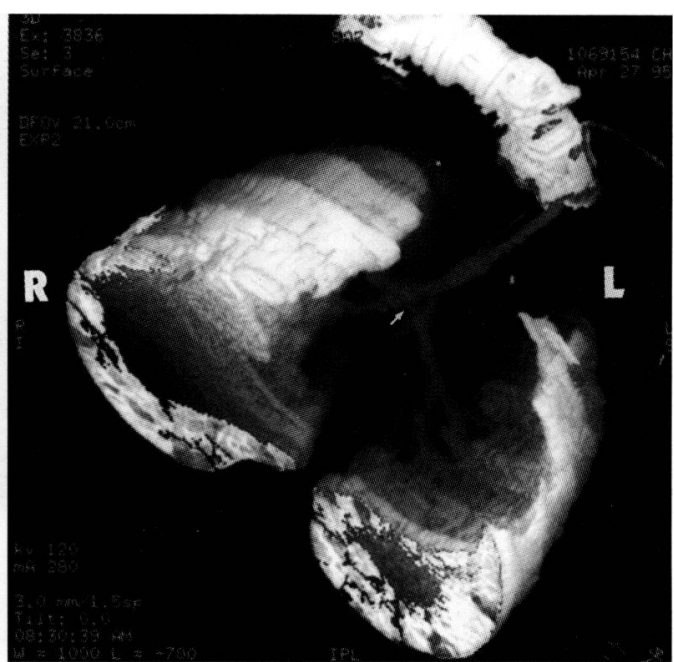

Figure 3 Right anterior oblique view of $3 D-C T$ image of the lungs in a four year old patient with chronic bronchopulmonary dysplasia and cardiomyopathy (patient 3). The arrow indicates ragged contour of the trachea in the region where surgery was done to repair tracheal stenosis. No disruption of the integrity of the entire tracheobronchial tree is observed.

repair (fig 3A and B). The patient subsequently underwent heart catheterisation which attributed her respiratory deterioration to cardiac dysfunction. Inotropic support with dobutamine was started and the patient died after three weeks of treatment.
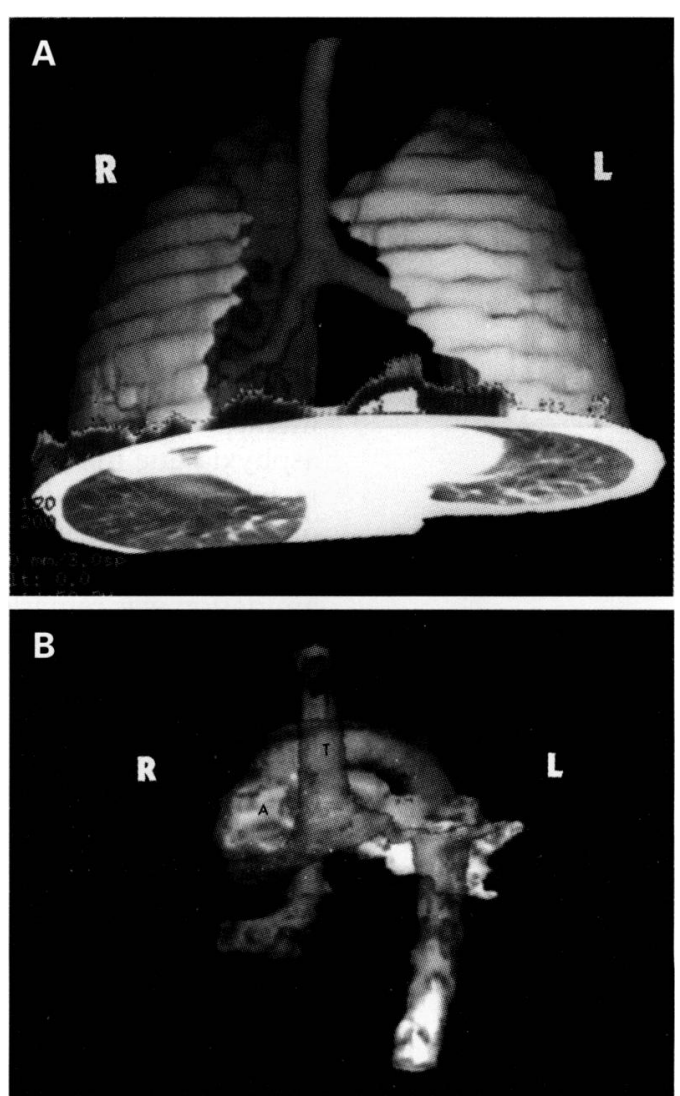

Figure 4 (A) Anterior view of 3D-CT image of the lungs in a three year old patient with Marfan's disease. The tracheobronchial tree configuration is not disrupted. (B) Further removal of intrathoracic tissue layers by changing density threshold levels to delineate the aortic contour. Mild dilation of the aortic root is seen $(A)$ with no disruption in the trachea $(T)$. 


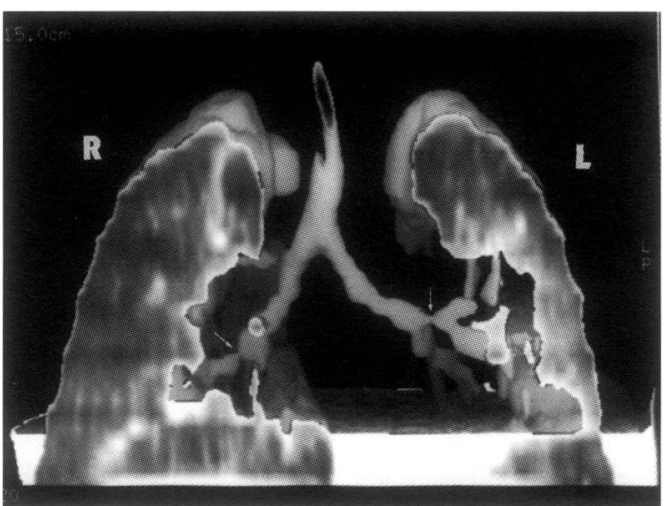

Figure 5 Anterior view of 3D-CT scan of the lungs in a six month old infant with recurrent episodes of respiratory failure. Note the narrow segments of the bronchi of the left upper lobe and the right middle lobe (arrows).

PATIENT 4

A three year old patient with Marfan's disease and a known dilation of the ascending aorta was admitted to the paediatric intensive care unit because of severe bronchospasm. The medical history was remarkable for frequent episodes of bronchospasm since the age of six months which were deemed to be secondary to asthma. The lack of a family history of allergic diseases did not support allergy as the aetiology for his bronchospasm. A 3D-CT scan of his chest was performed which revealed normal configuration of the tracheobronchial tree with only mild aortic root dilation (fig $4 \mathrm{~A}$ and $\mathrm{B}$ ). The patient was encouraged to continue with his anti-asthma medications.

\section{PATIENT 5}

A six month old baby who was admitted to the paediatric intensive care unit in severe respiratory distress and required endotracheal intubation and mechanical ventilation. His past medical history was remarkable for severe birth asphyxia due to meconium aspiration resulting in brain damage. He had had many episodes of aspiration pneumonitis due to inadequate protective airway reflexes. Physical examination was remarkable for diffuse wheezing over the lungs and hypertonia of his extremities. His chest radiograph revealed hyperinflation of the right lung and inadequate aeration of the left lung with left upper and lower lobe atelectases. A barium study through his gastrostomy tube revealed a major gastrooesophageal reflux to the level of the hypopharynx. A 3DCT scan showed narrow segments in the left upper lobe and right middle lobe bronchi (fig 5). Bronchoscopy revealed narrow segments in the left and right main bronchi around the orifices of the left upper lobe bronchus and the right middle lobe bronchus. These segments dilated to normal size with $15 \mathrm{~cm} \mathrm{H}_{2} \mathrm{O}$ of distending pressure and stayed open with a pressure of $10 \mathrm{~cm} \mathrm{H}_{2} \mathrm{O}$. A gastric fundoplication and tracheostomy was performed and chronic administration of $10 \mathrm{~cm} \mathrm{H} \mathrm{H}_{2} \mathrm{O}$ continuous positive airway pressure (CPAP) was initiated.

\section{Discussion}

The five patients described here had an underlying respiratory disorder that could be explained by a possible airway obstruction. In the first patient the combination of tetralogy of Fallot and absent pulmonary valve is known to be associated with airway compression by the aneurysmal dilation of the pulmonary artery. ${ }^{7}$ These patients may require more than one surgical procedure to relieve the compression and the function of their respiratory system may remain chronically compromised. Following heart catheterisation, the 3D-CT scan was a quick confirmatory study in this patient which delineated the airway segments compressed by the dilated pulmonary artery. Since this clinical entity is well known, we felt that the 3D-CT images were sufficient to confirm the clinical diagnosis and no further invasive procedures were required before surgery.

The 3D-CT scan in three of our other patients who presented with frequent episodes of respiratory distress due to lower airway obstruction and were refractory to treatment with bronchodilators provided valuable information as to whether or not bronchoscopy was indicated. In two cases (patients 2 and 5) the 3D-CT scan conspicuously delineated stenotic segments of the tracheobronchial tree which were subsequently diagnosed as segmental bronchomalacia by bronchoscopic examination. Bronchoscopy was also used to determine the degree of distending pressure that adequately dilated these segments and maintained them open. Bronchopulmonary dysplasia ${ }^{8}$ and recurrent episodes of aspiration pneumonitis, respectively, were assumed to be the aetiology for this clinical phenomenon. However, in patient 4 a normal configuration of the tracheobronchial tree was observed on the 3D-CT scan and thus bronchoscopy was prevented. Severe asthma was considered to be the cause for his episodes of bronchospasm. In patient 3, who had undergone a surgical repair of the trachea at an earlier age, the 3D-CT scan was also used to rule out restenosis of the intrathoracic airway as the reason for her deterioration without requiring bronchoscopy.

The importance of adequate imaging of the paediatric tracheobronchial tree for the investigation of possible obstruction has not been emphasised in the paediatric emergency and critical care medicine literature. The use of high voltage added-filtration radiography is cheap and helpful, yet not sensitive enough to detect changes in very narrow airways. ${ }^{9}$ Bronchoscopy is a useful diagnostic modality, but the passage of the bronchoscope may be impossible or may not be advisable through severely stenotic segments of the airway. ${ }^{10}$ Tracheobronchography in such cases can delineate the overall configuration of the tracheobronchial tree, but can also trigger an acute inflammatory process of the airways which may lead to death. ${ }^{11}$ Thus, fast and safe new imaging techniques should be appreciated if proved to be beneficial. Ultrafast cinetomography has been used to study infants and children with airway obstruction by displaying dynamic changes of the airway calibre during respira- 
tion. $^{12}$ This technique provides good anatomical detail of the airway and may demonstrate the dynamic collapse in cases of tracheomalacia and bronchomalacia. Our experience with 3D-CT scanning in these patients has also been valuable.

The 3D-CT scan is simply a computerised image of the axially acquired CT information. It does not therefore add new information but displays cross-sectional anatomy in a multiplanar manner. ${ }^{13}$ The three dimensional reconstructed images help physicians to visualise and understand the complex anatomical relationship within the thorax better. Moreover, the software used is capable of creating an image at any arbitrary orientation which may be particularly useful for surgical planning. ${ }^{14}$ In patients where the clinical diagnosis is well known, such as in the first patient with tetralogy of Fallot and absent pulmonary valve, the 3DCT images may be sufficient to determine the extent of the airway pathology and whether or not surgery is indicated. In other patients who present with frequent episodes of wheezing and in whom reactive airway disease is uncertain ${ }^{1}$ the $3 \mathrm{D}-\mathrm{CT}$ scan can be used as a non-invasive diagnostic tool for ruling out intrathoracic airway obstruction.

In summary, 3D-CT images of the chest in patients with suspected intrathoracic airway obstruction provide useful information. Negative findings on the 3D-CT scan may eliminate the need for other invasive diagnostic procedures such as bronchoscopy and bron- chography. However, when the 3D-CT images indicate a possible tracheobronchial obstruction, further diagnostic investigation may still be required.

1 Sagy M, Silver P, Nimkoff L, Zahtz G, Amato JJ, Bierman FZ. Pediatric intrathoracic large airway obstruction: diagnostic and therapeutic considerations. Pediatr Emerg Care 1994;10:351-8.

2 Costello P. Thoracic helical CT. Radiographics 1994;14: 913-8.

3 Brink JA, Heikn JP, Wang G, McEnergy KW, Schleuter FJ, Vannier MW. Helical CT: principles and technical consideration. Radiographics 1994;14:887-93

4 Manson D, Babyn P, Filler R, Holowka S. Three-dimensional imaging of the pediatric trachea in congenital tracheal stenosis. Pediatr Radiol 1994;24:175-9.

5 Newmark GM, Conces DJ Jr, Kopecky KK. Spiral CT evaluation of the trachea and bronchi. $\mathcal{F}$ Comput Assist Tomogr 1994;18:552-4.

6 Stern RL, Cline HE, Johnson GA, Ravin CE. Three-dimensional imaging of the thoracic cavity. Invest Radiol 1989;24:282-8.

7 Watterson KG, Malm TK, Karl TR, et al. Absent pulmonary valve syndrome: operation in infants with airway obvalve syndrome: operation in infants with
struction. Ann Thorac Surg 1992;54:1116-9.

8 Miller RW, Woo P, Kellman RK, Slagle T. Tracheobronchial abnormalities in infants with bronchopulmonary dysplasia. f Pediatr 1987;779-82.

9 Hernandez RJ, Tucker GF. Congenital tracheal stenosis: role of CT and high kV films. Pediatr Radiol 1987;17: 192-6.

10 Hancock BJ, Wiseman NE. Tracheobronchial injuries in children. F Pediatr Surg 1991;26:1316-9.

11 Idriss FS, Deleon SY, Ilbawi MN, Gerson CR, Tucker GF, Holinger L. Tracheoplasty with pericardial patch for extensive tracheal stenosis in infants and children. $\mathcal{F}$ Thorac Cardiovasc Surg 1984;88:527-36.

12 Newth CJ, Lipton MJ, Gould RG, Stretton M. Varying tracheal cross-sectional area during respiration in infants and children with suspected upper airway obstruction by computed cinetomography scanning. Pediatr Pulmono 1990;9:224-32

13 Vock P, Soueck M, Daepp M, Kalender WA. Lung: spiral volumetric $C T$ with single-breath-hold technique. Radiology 1990;176:864-7.

14 Brody AS, Kuhn JP, Seidel FG, Brodsky LS. Airway evaluation in children with use of ultrafast CT: pitfalls and recommendations. Radiology 1991;178:181-4. 